\title{
Editorial
}

\section{Walking the talk: clinic and our values}

Elaine Hall

Northumbria University, UK

Elaine.Hall@northumbria.ac.uk

Is clinical legal education an inherently moral undertaking? In this issue of the IJCLE, we have three papers linked by their focus on the underpinning values of CLE: whether we can transmit our values through the clinic experience; whether we can most effectively teach ethics through clinic and whether our commitment to and belief in clinic should be expressed by bringing it from the margins to the centre of legal education.

There is a common perception amongst educators that we transmit our values tacitly through the curriculum and that, by providing students with a particular kind of learning experience, we create in them specific dispositions. The logical formula appears to run:

1. We embed the values in the structure of the curriculum (e.g. by running a pro bono clinic)

2. We engage students in the activity

3. They absorb the values and become active agents pursuing those values in future activities. 
Once written down in this way, the potential for diversion from this programme becomes more apparent, not least the likelihood that

We had the experience but missed the meaning,

And approach to the meaning restores the experience

In a different form, (T.S Eliot).

In his paper, drawing on empirical research conducted at Northumbria University, Paul McKeown reflects on the evidence for 'values transfer' and looks critically both at the complex ecology of what students might take from experiential learning in a capstone ${ }^{1}$ clinic and how that data can be captured.

Anna Cody takes up the baton in her discussion of the teaching of ethics. Her paper provides a detailed engagement with what constitutes ethical and professional identity, developing a social constructivist frame for understanding how ethical cultures are formed and sustained. She develops this into an argument for 'applied ethics' teaching through a clinical element within the curriculum and provides a detailed analysis of how this operates in practice for students, supervisors and curriculum designers.

Finally, Neil Gold gathers together many years of clinical education experience to give an extended reflection on the untapped potential of clinic. Using a detailed analysis of educational standards, assessment and learning outcomes, he challenges

\footnotetext{
${ }^{1}$ A clinic experience placed at the end of a degree programme in which the prior academic and skills learning is operationalised.
} 
the marginal status of clinic within the legal curriculum, advancing the argument not just that CLE can deliver all the learning outcomes but that it is best suited to do so.

These debates continue, are enriched by more participants and on that note I would like to draw your attention to a series of upcoming events. Firstly, the forthcoming European Network for Clinical Legal Education (ENCLE) workshop which is being held at Northumbria University on $15^{\text {th }}$ and $16^{\text {th }}$ April 2015 and which is supported by the Open Society Initiative for Europe (OSIFE). The workshop will aim to generate discussion, through themed sessions, as to how clinicians can prepare their students for a clinical experience. Delegates will be able to share their own ideas and experiences and learn from others. Session facilitators will be drawn from a range of jurisdictions to enhance the discussion and draw out ideas for best practice ${ }^{2}$.

On $4^{\text {th }}$ June 2015 Northumbria will host an interactive seminar and discussion forum, Problematising Assessment in Clinical Legal Education ${ }^{3}$, featuring Professor Beryl Blaustone (City University of New York); Professor Jose Garcia Anon (Valencia University); Richard Grimes (University of York); Professor Judith McNamara (Queensland University of Technology) and Cath Sylvester (Northumbria University).

\footnotetext{
${ }^{2}$ For details of how to apply, please contact ustege@iuctorino.it or paul.mckeown@northumbria.ac.uk

${ }^{3}$ https://www.northumbria.ac.uk/about-us/academic-departments/northumbria-law-school/lawresearch/legal-education-and-professional-skills/problematising-assessment-in-clinical-legal-education/
} 
After that, we look forward to the next IJCLE conference, held jointly with the Global Alliance for Justice Education at Anadolu University in Eskişehir, Turkey between $22^{\text {nd }}$ and $28^{\text {th }}$ of July, 2015 (http://www.gaje.org/8th-worldwide-conference/). We are delighted to have contributions in the IJCLE stream addressing:

1. The Map for Learning Law: clinic and curriculum design papers considering the place of clinics in the wider law curriculum, the connections between legal knowledge and skills taught before, during and after the clinical experience and the specific goals of the clinic.

2. Collaborative Learning: comparative perspectives on clinic

papers involving two or more settings, focusing on how differences of context and ecology have shaped specific elements of practice locally as well as linking core practice and beliefs about clinic, education and outcomes for students and clients.

3. How do we know?: research, evidence and impact in clinical education

papers focusing specifically on methods of evaluation and the nature of the evidence that we use to understand and assess our clinics, representing a range of philosophical and methodological approaches.

Please let me know about CLE events and I will publicise them here - do bear in mind our approximate publication dates of February, July and November.

I hope to see many of you at these events! 
References

Eliot, T.S. (1941) The Dry Salvages. Published in the New English Weekly, collected in The Four Quartets (1943) New York: Harcourt 